17 Takagi T, Kondo T, Tajima T, Campbell SC, Tanabe K. Enhanced computed tomography after partial nephrectomy in early postoperative period to detect asymptomatic renal artery pseudoaneurysm. Int. J. Urol. 2014; 21: 880-5.

18 Omae K, Kondo T, Takagi T et al. Renal sinus exposure as an independent factor predicting asymptomatic unruptured pseudoaneurysm formation detected in the early postoperative period after minimally invasive partial nephrectomy. Int. J. Urol. 2015; 22: 356-61.
19 Morita S, Tajima T, Yamazaki H et al. Early postoperative screening by contrast-enhanced $\mathrm{CT}$ and prophylactic embolization of detected pseudoaneurysms prevents delayed hemorrhage after partial nephrectomy. J. Vasc. Interv. Radiol. 2015; 26: 950-7.

20 Kondo T, Takagi T, Morita $\mathrm{S}$ et al. Early unclamping might reduce the risk of renal artery pseudoaneurysm after robot-assisted laparoscopic partial nephrectomy. Int. J. Urol. 2015; 22: 1096-102.

\title{
Editorial Comment
}

\section{Editorial Comment to Robot-assisted laparoscopic versus open partial nephrectomy in patients with chronic kidney disease: A propensity score-matched comparative analysis of surgical outcomes}

Partial nephrectomy (PN) is mandatory for the treatment of clinical T1 renal tumors in patients with chronic kidney disease. In the past decade, robot-assisted PN (RAPN) has been increasingly used for its technical precision in addition to the undebatable benefits of minimally-invasive surgery.

In the present article, Takagi et al. analyzed the surgical and functional outcomes of the open and the robotic approach in the setting of patients with pre-existing stage 3 chronic kidney disease by a matched-paired comparison accounting for age, sex, Easter Cooperative Oncology Group performance status, body mass index, comorbidities, American Society of Anesthesiologists score and tumor nephrometry RENAL score. ${ }^{1}$ The authors conclude that the patients undergoing RAPN have a significantly lower rate of overall complications and bleeding, and a higher rate of immediate postoperative renal function (RF) preservation compared with those who underwent open $\mathrm{PN}$, whereas delayed RF recovery 3-6 months after surgery was comparable between the two approaches.

The work joins a chorus of research that suggests RAPN can safely reply the classical open procedure, even in challenging cases as those where the preservation of postoperative RF is of utmost importance. In this clinical setting, avoiding or reducing functional damage related to surgical modifiable parameters plays a fundamental role. In this regard, our understanding about the decline in renal function after PN has advanced considerably, and emerging evidence has showed that the volume of healthy vascularized preserved parenchyma after tumor resection and renorraphy along with ischemia time have a significant impact on RF recovery.

Types of resection technique; that is, enucleation and enucleoresection, are just mentioned in this article, but neither were stratified between the two surgical approaches nor analyzed as potential postoperative RF determinants. Indeed, wider resection margins do not lead to improved cancer control, but might affect the quantity of parenchyma preserved and consequently RF. ${ }^{2}$ In this regard, for future studies, we should aim to standardize resection technique definitions through a comprehensive reporting model. ${ }^{3}$

Partial nephrectomy is not devoid of complications, and the present study confirms the lower morbidity of the robotic approach in comparison with the open counterpart, especially limiting the low-grade complication rate and thus the overall complication rate, leading to a shorter length of stay and faster recovery. ${ }^{4,5}$ In conclusion, in this clinical setting, according to the study by Takagi et al., RAPN can attain better early postoperative clinical and functional outcomes than open partial nephrectomy. Furthermore, RAPN is a less invasive and safer approach, offering lower intraoperative bleeding and postoperative complications than the open approach in agreement with the current literature on this topic. Further studies are required to confirm the functional benefits of RAPN in comparison with the other approaches adjusting for the several modifiable and unmodifiable factors that have a relevant impact on PN functional outcomes.

Andrea Minervini M.D., Ph.D., Andrea Mari M.D. and Marco Carini M.D. Department of Urology, Careggi Hospital, University of Florence, Florence, Italy andreamine@libero.it

DOI: $10.1111 /$ iju. 13388

\section{Conflict of interest}

None declared.

\section{References}

1 Takagi T, Kondo T, Tachibana H et al. Robot-assisted laparoscopic versus open partial nephrectomy in patients with chronic kidney disease: a propensity score-matched comparative analysis of surgical outcomes. Int. J. Urol. 2017; 24: 505-10.

2 Simone G, Gill IS, Mottrie A et al. Indications, techniques, outcomes, and limitations for minimally ischemic and off-clamp partial nephrectomy: a systematic review of the literature. Eur. Urol. 2015; 68: 632-40.

3 Minervini A, Carini M, Uzzo RG, Campi R, Smaldone MC, Kutikov A. Standardized reporting of resection technique during nephron-sparing surgery: the surface-intermediate-base margin score. Eur. Urol. 2014; 66: 803-5.

4 Ficarra V, Minervini A, Antonelli A et al. A multicentre matched-pair analysis comparing robot-assisted versus open partial nephrectomy. BJU Int. 2014; 113: 936-41.

5 Porpiglia F, Mari A, Bertolo R et al. Partial nephrectomy in clinical T1b renal tumors: multicenter comparative study of open, laparoscopic and robot-assisted approach (the RECORd Project). Urology 2016; 89: 45-51. 